\title{
Computational Studies and Peptidomimetic Design for the Human p53-MDM2 Complex
}

\author{
Haizhen Zhong and Heather A. Carlson* \\ Department of Medicinal Chemistry, College of Pharmacy, University of Michigan, Ann Arbor, Michigan
}

\begin{abstract}
The interaction between human p53 and MDM2 is a key event in controlling cell growth. Many studies have suggested that a p53 mimic would be sufficient to inhibit MDM2 to reduce cell growth in cancerous tissue. In order to design a potent p53 mimic, molecular dynamics (MD) simulations were used to examine the binding interface and the effect of mutating key residues in the human p53-MDM2 complex. The Generalized Born surface area (GBSA) method was used to estimate free energies of binding, and a computational alanine-scanning approach was used to calculate the relative effects in the free energy of binding for key mutations. Our calculations determine the free energy of binding for a model p53-MDM2 complex to be $-7.4 \mathrm{kcal} / \mathrm{mol}$, which is in very good agreement with the experimentally determined values (-6.6$-8.8 \mathrm{kcal} / \mathrm{mol})$. The alanine-scanning results are in good agreement with experimental data and calculations by other groups. We have used the information from our studies of human p53-MDM2 to design a $\beta$-peptide mimic of p53. MD simulations of the mimic bound to MDM2 estimate a free energy of binding of $-8.8 \mathrm{kcal} / \mathrm{mol}$. We have also applied alanine scanning to the mimic-MDM2 complex and reveal which mutations are most likely to alter the binding affinity, possibly giving rise to escape mutants. The mimic was compared to nutlins, a new class of inhibitors that block the formation of the p53MDM2 complex. There are interesting similarities between the nutlins and our mimic, and the differences point to ways that both inhibitors may be improved. Finally, an additional hydrophobic pocket is noted in the interior of MDM2. It may be possible to design new inhibitors to take advantage of that pocket. Proteins 2005;58:222-234.

$\odot 2004$ Wiley-Liss, Inc.
\end{abstract}

Key words: HDM2; $\beta$-peptide; molecular dynamics simulation; free energy calculation; escape mutants; inhibitor; protein mimic; AMBER

\section{INTRODUCTION}

The 53-kDa phosphoprotein p53 plays a key role in maintaining the genomic integrity of cells. In response to DNA damage and other types of stress stimuli, p53 causes cell-cycle arrest ${ }^{1}$ or activates apoptosis. ${ }^{2,3}$ In normal cells, p53 is held in check until needed by MDM2 (the murine double-minute clone 2 , more appropriately termed human double-minute clone 2, or HDM2, for our system). ${ }^{4}$ Detrimental mutations of p53 are common mechanisms for the loss of p53 wild-type activity in cancer cells. ${ }^{5}$ But another important mechanism is overexpression of MDM2, which leads to constitutive inhibition of p53; this is commonly seen in cancerous cells containing wild-type (WT) p53. ${ }^{6-9}$

Because of its importance in cancer development, the p53-MDM2 complex is a target for anticancer drug design. It has been shown that a p53 homologue is sufficient to induce p53-dependent cell death in cells overexpressing MDM2. ${ }^{10}$ It has been shown that a peptide as short as 6 residues could bind to MDM2 in the same manner, ${ }^{11}$ and medicinal chemistry modifications to that 6-residue peptide dramatically increased its inhibitory activity. ${ }^{12}$ Small p53 mimics would be expected to disrupt the p53-MDM2 complex, consequently liberating p53 to initiate cell-cycle arrest or apoptosis. This anti-MDM2 approach has been shown to reestablish p53 activities in malignant tissues with amplified MDM2 genes. The most recent advance has been the discovery of nutlins: small, multicyclic, hydrophobic antagonists that associate to MDM2 within the binding cleft that recognizes p53. ${ }^{13}$ These inhibitors are able to induce apoptosis via the p53 pathway in cancer cells.

The first calculation of the p53-MDM2 system was a 400-ps molecular dynamics (MD) simulation by Massova and Kollman. ${ }^{14}$ In that study, a computational alaninescanning method was introduced to evaluate the individual contributions to the binding free energy for each residue in the key helix of p53. In order to design an effective p53 mimic as an inhibitor of human MDM2, it is important to understand the p53-MDM2 interaction at the atomic level. More importantly, the design process will benefit from understanding the influence of MDM2's individual residues on the free energy of binding. To achieve these goals, we have conducted MD simulations and

Grant sponsor: University of Michigan. Grant sponsor: National Institutes of Health; Grant number: GM65372. Grant sponsor: Beckman Young Investigator Program.

The Supplementary Materials referred to in this article can be found at http://www.interscience.wiley.com/jpages/0887-3485/suppmat/ index.html

*Correspondence to: Heather A. Carlson, Department of Medicinal Chemistry, College of Pharmacy, University of Michigan, 428 Church St., Ann Arbor, MI 48109-1065. E-mail: carlsonh@umich.edu

Received 10 March 2004; Accepted 23 June 2004

Published online 25 October 2004 in Wiley InterScience (www.interscience.wiley.com). DOI: 10.1002/prot.20275 
computational alanine scanning for the p53-MDM2 complex, focusing on MDM2.

We have used the information from our simulations to design a mimic of p53 based on $\beta$-proline (an isomer of $\alpha$-proline). These designs build off our previous ab initio calculations of the conformational properties of $\beta$-proline helices. ${ }^{15,16} \beta$-Peptides are amino acids with the carboxylic acid functionality at $\mathrm{C} \beta$ rather than $\mathrm{C} \alpha$. The difference in the chiral center allows $\beta$-peptides to resist hydrolysis by proteases in the body, giving them different absorption, distribution, metabolism, and excretion (ADME) properties than $\alpha$-peptides. ${ }^{17-19}$ Biomimetic polymers like these hold promise for new biomaterials and therapeutics, ${ }^{20-22}$ and this study shows how they may be used in structurebased drug discovery to target protein-protein recognition events in cancer cells.

\section{COMPUTATIONAL METHODS}

The MD simulations and computational alanine-scanning calculations were performed with the AMBER6. $0^{23}$ suite of programs using the AMBER94 force field ${ }^{24}$ (the $\beta$-proline inhibitor required additional ester parameters from AMBER99; see supplemental information). The crystal structure of the human p53-MDM2 complex [Protein Data Bank code: $1 \mathrm{YCR}]^{25}$ was used. In the crystallization study, residues 17-125 of human MDM2 were used, but only residues 25-109 were resolved in the crystal structure. In our simulations, the amino terminus (E25) and the carbonyl terminus (V109) of MDM2 were capped with an acetyl group (ACE) and an $N$-methyl group (NME), respectively. Similarly, the N-terminus of the p53 helix (E17) was capped with ACE group because of the absence of electron density for residues 15 and 16 of p53 (the 15-29 stretch of p53 was used in the crystallization). Our simulations used a 13-residue stretch of p53 (Ac-ETFSDLWKLLPEN-COO-) in complex with MDM2 to be consistent with the crystal structure determination. Hydrogens were added to the protein using AMBER.

\section{Molecular Dynamics}

We performed the MD simulations on 5 different systems: the p53-MDM2 complex, p53 alone in solution, MDM2 alone, MDM2 complexed with our $\beta$-proline mimic, and the mimic alone. All systems were solvated by cubic boxes of TIP3P water, ${ }^{26}$ which extended at least $12 \AA$ away from any given protein atom. The MDM2 system had a net charge of +5 , so 5 chloride ions were added to neutralize the system. In a similar way, 2 chloride ions were added to neutralize the p53-MDM2 system, and 3 sodium ions were added to the p53 system. Our mimic is net neutral and required no counterions. The mimic-MDM2 complex required 5 chloride ions. We used the Poisson-Boltzmann electrostatics module of the Molecular Operating Environment package (MOE) ${ }^{27}$ to visualize the field at $8 \AA$ and 10 $\AA$ from the protein surface. We placed the ions at the most charged locations on those surfaces.

The SANDER_CLASSIC module of the AMBER6.0 package was used for the MD simulations. The particle mesh Ewald method ${ }^{28}$ was used to treat the long-range electro- statics. Periodic boundary conditions and a 10-Å cutoff for nonbonded van der Waals (vdw) interactions were applied. All bonds involving hydrogen atoms were constrained using the SHAKE algorithm. Constant temperature and pressure $(300 \mathrm{~K} / 1 \mathrm{~atm})$ were maintained. A 2 -fs timestep was used to integrate the equations of motion.

The following equilibration protocol was applied to all systems: First, the water molecules were energy-minimized for 500 steps using the Steepest Descent algorithm, while holding the solute frozen, followed by minimization of the protein systems (including the counterions) for 500 steps, while holding the water frozen. Subsequently, the whole system was subjected to 500 steps of minimization to remove close contacts and to relax the system. Finally, the whole system was subjected to a gradual temperature increase from $10 \mathrm{~K}$ to $300 \mathrm{~K}$ in 6 intervals over $30 \mathrm{ps}$. The whole system was then equilibrated for $100 \mathrm{ps}$. After this, $2 \mathrm{~ns}$ of averaging was conducted. Coordinates were saved every $1 \mathrm{ps}$ for a total of 2000 snapshots. The resulting trajectories were analyzed using the PTRAJ and CARNAL modules of AMBER6.0.

\section{Free Energy Calculations}

The GBSA (molecular mechanics/Generalized Born surface area) method $^{29,30}$ was used to calculate the free energy of binding $\left(\Delta \mathrm{G}_{\mathrm{bind}}\right)$. One can estimate the binding free energies to estimate the binding of p53 or the mimic to MDM2 using the following equations:

$$
\begin{aligned}
\Delta \mathrm{G}_{\text {bind }}=\left\langle\mathrm{G}_{\text {water }}(\text { complex })\right\rangle \\
-\left[\left\langle\mathrm{G}_{\text {water }}(\mathrm{MDM} 2)\right\rangle+\left\langle\mathrm{G}_{\text {water }}(\text { ligand })\right\rangle\right],
\end{aligned}
$$

where $\left\langle G_{w a t e r}\right\rangle$ is the average free energy of the system in solution calculated by the following:

$$
\begin{gathered}
\left\langle\mathrm{G}_{\mathrm{water}}\right\rangle=\left\langle\mathrm{E}_{\text {gas }}\right\rangle+\left\langle\mathrm{G}_{\text {solvation }}\right\rangle-\mathrm{T}\langle\mathrm{S}\rangle \\
E_{\text {gas }}=E_{\text {bond }}+E_{\text {angle }}+E_{\text {torsion }}+E_{\text {vdw }}+E_{\text {electrostatic }} \\
G_{\text {solvation }}=G_{G B}+G_{\text {nonpolar, }},
\end{gathered}
$$

where $\mathrm{E}_{\text {gas }}$ is the sum of the bond, angle, torsion, vdw, and electrostatic terms from the conformations saved from the MD simulation. The "gas" notation indicates that the total energy term does not include the interaction between the solutes and the surrounding explicit water molecules or ions from the $\mathrm{MD}$ simulation. The absolute energy, $\mathrm{E}_{\text {gas }}$, was calculated using the ANAL module in the AMBER6.0 package. All energy components and solvation contributions were calculated using trajectories from 3 systems (the ligand-MDM2 complex, the unbound MDM2, and the unbound ligand, where the ligand can be the p53 helix or our $\beta$-proline mimic).

The free energy of solvation $\left(\mathrm{G}_{\text {solvation }}\right)$ includes polar $\left(\mathrm{G}_{\mathrm{GB}}\right)$ and nonpolar contributions $\left(\mathrm{G}_{\text {nonpolar }}\right)$. The polar electrostatic contribution was estimated using Generalized Born theory (employing the program $\mathrm{GB}^{31}$ in the AMBER package). The nonpolar contribution to the solvation free energy was estimated according to the equation, $\mathrm{G}_{\text {nonpolar }}=\gamma^{*}$ SASA $+\beta$, where SASA is the solventaccessible surface area calculated by the program MSMS 
and the default values of $\gamma$ and $\beta$ were used $(\gamma=0.00542$ $\mathrm{kcal} / \AA^{2}$ and $\left.\beta=0.92 \mathrm{kcal} / \mathrm{mol}\right){ }^{32}$

The average entropy, $\langle\mathrm{S}\rangle$, for each system was calculated from 11 snapshots taken every $200 \mathrm{ps}$ from the 2-ns trajectory. The entropy was estimated using normal mode analysis (employing the NMODE module ${ }^{33,34}$ of AMBER). To prepare structures for the normal mode calculations, the water and counterions were removed from each snapshot, and the protein structure was energy-minimized. After minimization, the conformation was compared to the original snapshot; all resulting conformations had a rootmean-square deviation (RMSD) less than $2 \AA$ to the original snapshot, indicating that those structures were representative of the structures sampled in the MD simulations.

\section{Computational Alanine Scanning}

Key residues of MDM2 were chosen from the binding interface: L54, L57, I61, M62, Y67, Q72, F91, V93, H96, I99, and Y100. Each of these residues is within $4 \AA$ of p53 and the $\beta$-proline mimic. We calculated the relative change in free energy of binding $\left(\Delta \Delta \mathrm{G}_{\text {bind }}\right)$ for the alanine mutants of these residues in MDM2 using GBSA. The alanine mutants were created by truncating the key residue in the snapshots from the MD simulations of the WT MDM2ligand complexes. For this calculation, we followed the original protocol outlined by Massova and Kollman, ${ }^{14}$ which assumes that the entropy of the mutant is not significantly different than the WT and the components for $\Delta \Delta \mathrm{G}_{\mathrm{bind}}$ come only from the changes in $\left\langle\mathrm{E}_{\text {gas }}\right\rangle$ and $\left\langle\mathrm{G}_{\text {solvation }}\right\rangle . \Delta \Delta \mathrm{G}_{\text {bind }}$ was calculated by comparing the $\Delta \mathrm{G}_{\text {bind }}$ of the alanine mutant to the $\Delta \mathrm{G}_{\text {bind }}$ of the WT.

\section{RESULTS AND DISCUSSION Molecular Dynamics of p53-MDM2 Systems}

In order to examine the binding interface and estimate the free energy of binding for the p53-MDM2 complex, we performed 2-ns MD simulations on 3 different systems: the p53-MDM2 complex, the unbound MDM2, and the unbound p53. The potential energy of the protein systems over the course of the simulations is given in the supplemental information. The energies of the 3 systems indicate that all were stable over the 2 -ns simulations.

The stability of the simulations is further demonstrated in Figure 1, with the RMSD of the $\mathrm{C} \alpha$ atoms compared over the course of the simulation. The trajectory of the unbound MDM2 is quite stable over the timescale of the simulation, with an average RMSD less than $1.0 \AA$ [gray line in Figure 1(B)]. The RMSD of the p53-MDM2 complex was under $1.0 \AA$ during the first nanosecond, but a rise in the RMSD is observed between 1.0 and $1.7 \mathrm{~ns}$. As seen in Figure 1(A), the increase comes from the p53 helix in the p53-MDM2 complex. The $\mathrm{N}$-terminus of the $\mathrm{p} 53$ helix unwound slightly and sampled more conformational space in the section half of the simulation [Fig. 2(A)]. This terminus is partially unresolved in the crystal structure, so higher flexibility is expected.

For the unbound p53 system [the black line in Fig. 1(B)], the RMSD continues to increase over the simulation,

\section{A MD of Human p53-MDM2 Complex}

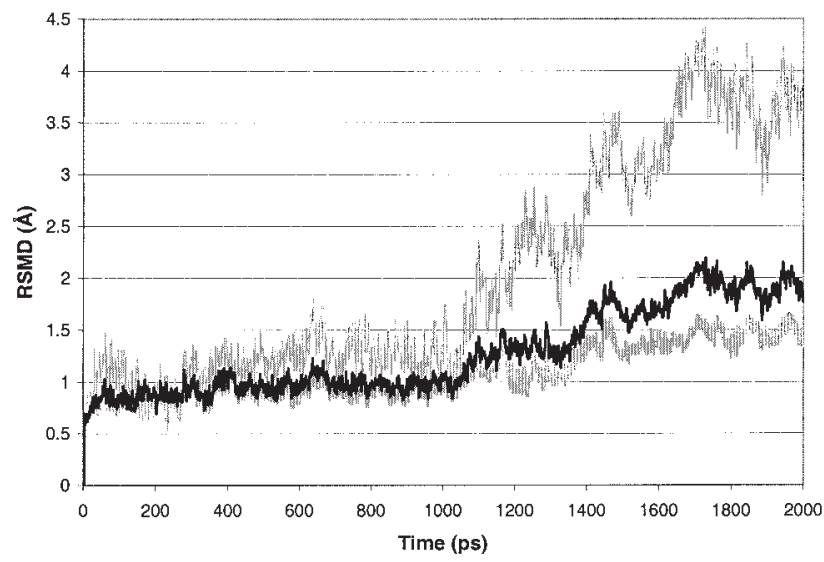

B Independent MD of Unbound p53 and MDM2

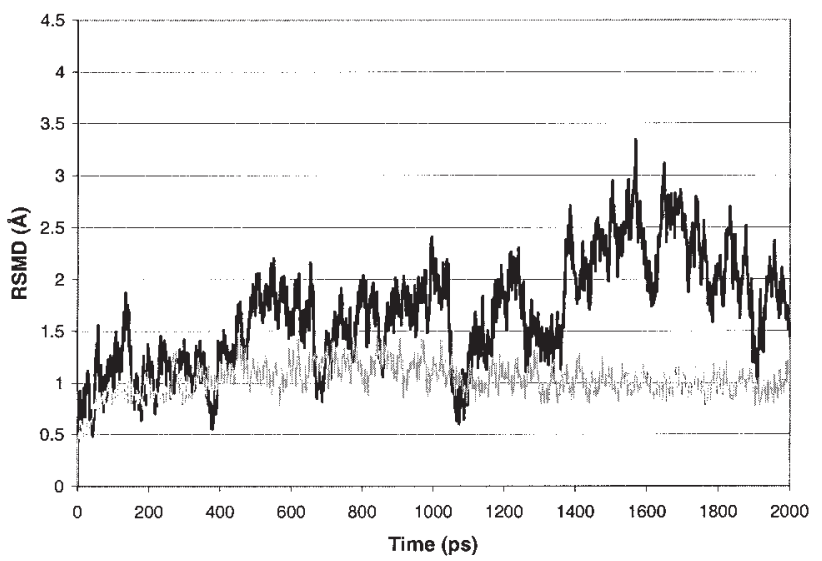

Fig. 1. (A) The RMSDs for the $\mathrm{C} \alpha$ atoms are provided for the complex of human p53-MDM2 (thick black line), MDM2 in the complex (thick gray line), and p53 in the complex (thin gray line). All 3 plots were calculated from an overlay of all $\mathrm{C} \alpha$ atoms in the complex. (B) The RMSDs for the $\mathrm{C} \alpha$ atoms in the simulations of unbound p53 (thick black line) and unbound MDM2 (thin gray line).

caused primarily by more motion in the side chains and the C-terminus. Massova and Kollman also observed a great deal of flexibility for the unbound p53 helix. ${ }^{14}$ It appears that the structure of the short p53 sequence is stabilized by the hydrophobic binding cleft of MDM2, and its helical structure is not as stable in solution [Fig. 2(B)]. Short peptides are often highly flexible in solution, and the p53 helix appears to follow this trend. The side chains of F19 and W23 swing freely over the 2-ns simulation [Fig. 2(B)]. The average RMSDs for the F19 and W23 side chains from the unbound p53 simulation are $2.5 \AA$ and 3.5 $\AA$, respectively. However, the F19 and W23 side-chains of p53 complexed to MDM2 sampled much less conformational space (average RMSD of $1.5 \AA$ and $1.2 \AA$, respectively). This suggests that the hydrophobic interaction between MDM2 and p53 helps to stabilize and maintain the p53 helix. The small degree of conformational sampling in the complex also suggests the importance of properly oriented aromatic groups for a successful steric complement to the MDM2 binding pocket. 


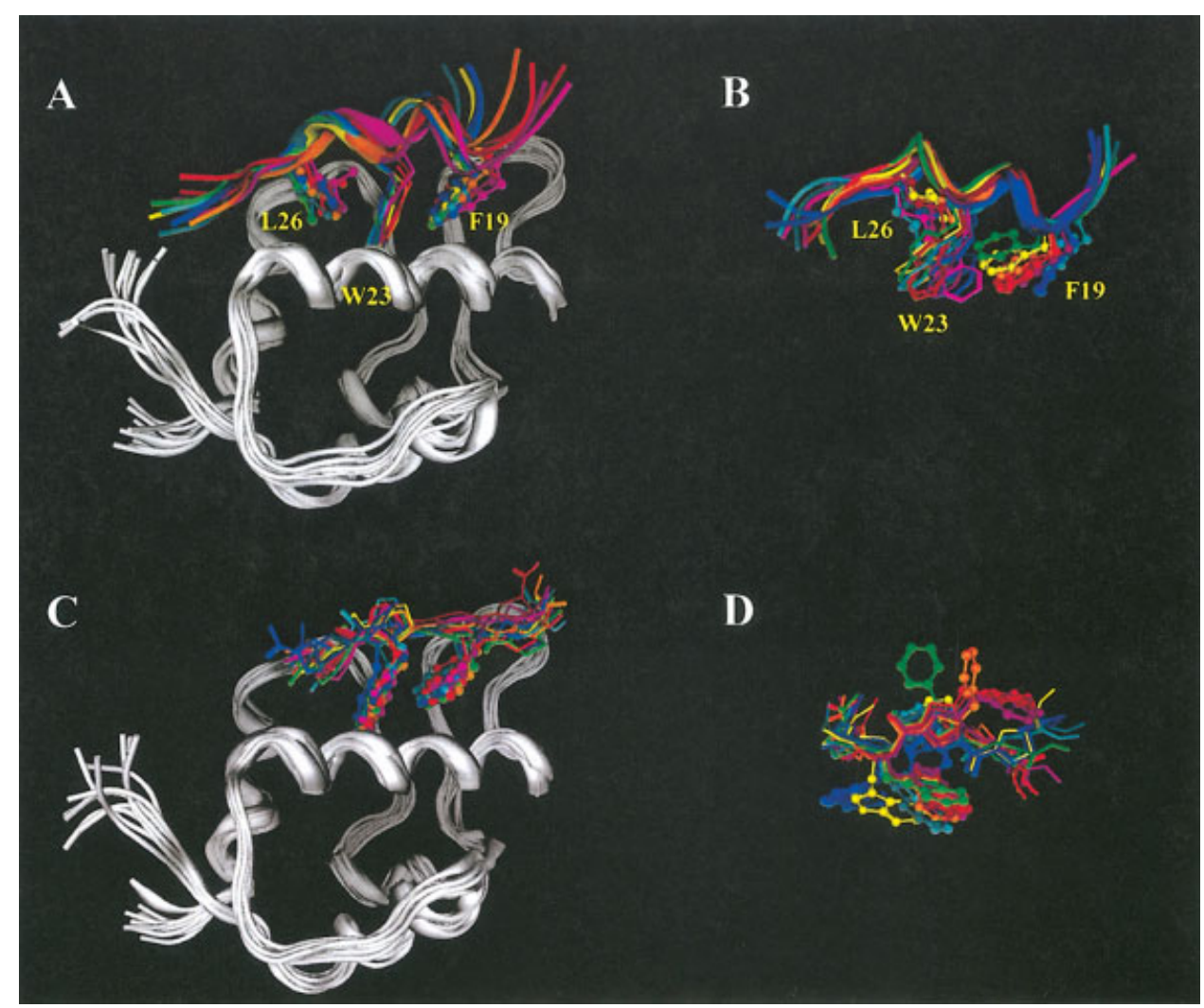

Fig. 2. Snapshots from the 2-ns MD simulations are color coded from early (dark blue and green) to middle (yellow) to late (red and purple) in the simulation. The backbone of MDM2 is shown in gray for all snapshots, and all figures are drawn to the same scale. (A) The conformational sampling of the backbone of p53-MDM2 with F19, W23, and L26 highlighted. (B) Structures of unbound p53 show that the hydrophobic groups have wide conformational sampling; p53 seems less stable in the absence of MDM2. (C) Conformational sampling of the mimic-MDM2 complex. (D) In the unbound mimic, the aromatic rings also show wide conformational sampling (atoms shown in ball-and-stick configuration), but the backbone is very stable (atoms shown in stick model).

\section{Free Energy Calculations}

The apparent $K_{\mathrm{d}}$ for the helix of p53 (residues 15-29 used in the crystal structure) is $600 \mathrm{n} M$ at $35^{\circ} \mathrm{C}(-8.8$ $\mathrm{kcal} / \mathrm{mol}){ }^{25}$ The $\mathrm{IC}_{50}$ for the binding of a sequence from human p53 (Ac-QETFSDLWKLLP-NH2) to MDM2 is 2-14 $\mu M$ as reported by Böttger et al. ${ }^{11}$ This $\mathrm{IC}_{50}$ range corresponds to a free energy of binding between -6.6 and $-7.8 \mathrm{kcal} / \mathrm{mol} .{ }^{14}$ The sequence that we used from the crystallographic study is slightly different, but we assume that the binding affinity should be similar. The contributions to the free energy of binding for the p53-MDM2 complex are listed in Table I and were obtained using the GBSA method. The electrostatic energy is balanced by the free energy of solvation, so it appears that the vdw energy drives the association. Our calculated $\Delta \mathrm{G}_{\mathrm{bind}}$ for $\mathrm{p} 53-$ MDM2 is $-7.4 \mathrm{kcal} / \mathrm{mol}$. This is in excellent agreement with related experimental values. Despite some conformational sampling of the p53 helix toward the end of $2 \mathrm{~ns}$ of $\mathrm{MD}$, the $\Delta \mathrm{G}_{\text {bind }}$ values are consistent across the simulation. It appears that last few residues in the $\mathrm{N}$-terminus of the p53 helix do not contribute as significantly to binding as residues that interact with the cleft.

\section{Entropic Component}

When using the GBSA method to calculate the binding free energy, careful protocol should be used when applying the NMODE method to estimate the entropic contribution (S). We adopted a different way to estimate entropy than was originally proposed. In the original presentation of the method, ${ }^{14}$ only the crystal structure was used to estimate the entropy. Since the energy components and the related free energy components are the average of all 2000 snapshots from the 2-ns simulations, it is more appropriate if we use several snapshots from the MD to calculate the entropy. Using all 2000 snapshots was prohibitively timeconsuming, so we used 11 snapshots (the equilibration structure plus structures from every $200 \mathrm{ps}$ ) to calculate an average entropy, $\langle\mathrm{S}\rangle$.

The original paper also presented a second approximation with respect to entropy. The conformations of the proteins in the crystal structure were used to estimate the entropy of the complex and also to estimate the entropies for MDM2 alone and for the p53 helix alone. We evaluated 2 methods to determine the average entropy of the unbound MDM2 and p53. Our first method was similar to the 
TABLE I. Energy Components and Free Energy of Binding for the Human p53-MDM2 Complex ${ }^{\mathrm{a}}$

\begin{tabular}{|c|c|c|c|c|c|c|c|}
\hline \multirow{2}{*}{$\begin{array}{l}\text { Contributions } \\
\text { (kcal/mol) }\end{array}$} & \multicolumn{2}{|c|}{ p53-MDM2 Complex } & \multicolumn{2}{|c|}{ Receptor (MDM2) } & \multicolumn{2}{|c|}{ Ligand (p53) } & \multirow[b]{2}{*}{$\Delta$} \\
\hline & average & std dev & average & std dev & average & std dev & \\
\hline $\mathrm{E}_{\text {electrostatic }}$ & -2927.0 & 88.8 & -2388.8 & 42.6 & -177.7 & 20.4 & -360.4 \\
\hline $\mathrm{E}_{\mathrm{vdw}}$ & -396.2 & 20.0 & -322.7 & 14.7 & -9.8 & 4.7 & -63.8 \\
\hline $\mathrm{E}_{\text {internal }}$ & 1681.5 & 28.3 & 1456.4 & 26.5 & 214.5 & 9.9 & 10.6 \\
\hline $\mathrm{E}_{\text {gas }}$ & -1641.7 & 100.4 & -1255.1 & 47.7 & 26.9 & 22.7 & -413.5 \\
\hline $\mathrm{G}_{\text {nonpolar }}$ & 45.7 & 1.3 & 42.5 & 0.6 & 13.4 & 0.3 & -10.2 \\
\hline $\mathrm{G}_{\mathrm{GB}}$ & -1232.2 & 82.6 & -1169.3 & 39.1 & -432.2 & 17.7 & 369.2 \\
\hline $\mathrm{G}_{\text {solvation }}$ & -1186.5 & 82.1 & -1126.8 & 38.8 & -418.7 & 17.7 & 359.0 \\
\hline $\mathrm{E}_{\text {gas }}+\mathrm{G}_{\text {solvation }}$ & -2828.2 & 33.6 & -2381.8 & 26.4 & -391.8 & 10.4 & -54.5 \\
\hline $\begin{array}{l}-\mathrm{TS}^{\mathrm{b}} \\
\Delta \mathrm{G}_{\mathrm{bind}}^{\mathrm{b}}\end{array}$ & -1108.5 & 5.3 & -970.6 & 2.9 & -185.0 & 2.9 & $\begin{array}{r}47.1 \\
-7.4\end{array}$ \\
\hline $\begin{array}{l}-\mathrm{TS}^{\mathrm{c}} \\
\Delta \mathrm{G}_{\text {bind }}{ }^{\mathrm{c}}\end{array}$ & -1108.5 & 5.3 & -971.4 & 4.7 & -177.6 & 2.0 & $\begin{array}{r}40.5 \\
-14.1 \\
\end{array}$ \\
\hline
\end{tabular}

${ }^{a}$ Any small discrepancies are due to rounding to the first decimal place.

${ }^{b}$ Entropy was estimated based on structures from the trajectory of the complex only. $\Delta \mathrm{G}_{\text {bind }}$ from experiments is between -6.6 and $-8.8 \mathrm{kcal} / \mathrm{mol}$. Our calculated $\Delta \mathrm{G}_{\text {bind }}$ of $-7.4 \mathrm{kcal} / \mathrm{mol}$ is in very good agreement with the experiments, indicating the reliability of the MM-GBSA method coupled with this approach to estimating the entropy.

${ }^{\mathrm{c}}$ Entropy was estimated based on separate trajectories of the complex, MDM2, and p53.

original methodology, because we estimated the entropy of the complex and components using only snapshots from the simulation of the p53-MDM2 complex. Using the first method, we were able to reach agreement with experiment and calculate $\Delta \mathrm{G}_{\text {bind }}$ to be $-7.4 \mathrm{kcal} / \mathrm{mol}$. The second method calculated each system's average entropy using snapshots from separate trajectories of the complex (p53MDM2), MDM2 alone in solution, and p53 alone. Using the second method, $\Delta \mathrm{G}_{\text {bind }}$ was calculated to be $-14.1 \mathrm{kcal} /$ mol. This is much higher than the experimental $\mathrm{IC}_{50}$ 's imply and would indicate incredibly tight binding. The second method appears to overestimate the entropy, but the cause is unclear. Independent sampling for the unbound components should be a more accurate procedure for estimating entropic changes upon binding.

Our use of independent trajectories for calculating the entropic change upon binding was not successful, but our use of longer sampling and an average entropy term appears to be an improvement over the original implementation by Massova and Kollman. ${ }^{14}$ They calculated a smaller free energy of binding for the human p53-MDM2 complex, between -4.5 and $-5.7 \mathrm{kcal} / \mathrm{mol}$ (still good agreement with the experimental values given the approximate nature of the methodology). Our higher $\Delta \mathrm{G}_{\text {bind }}$ may also be due to our use of a slightly different sequence for the p53 helix. We have also chosen to use the GB method to estimate electrostatics rather than the Poisson-Boltzmann electrostatics used by Massova and Kollman.

\section{Alanine Scanning Within the Binding Cleft of MDM2}

The alanine-scanning methodology is based on the assumption that replacing the original residue with an alanine will only introduce local changes and not cause a large conformational change to alter the binding mode. Also, the small local changes are assumed to have little effect on entropy, so the entropic term $(-\mathrm{T} \Delta\langle\mathrm{S}\rangle)$ for the wild-type and the mutants should cancel when calculating $\Delta \Delta \mathrm{G}_{\text {bind }}$. Therefore, $\Delta \Delta \mathrm{G}_{\text {bind }}$ is defined as $\Delta \mathrm{G}_{\text {bind }}(\mathrm{WT})-$ $\Delta \mathrm{G}_{\text {bind }}$ (Ala mutant), but is only calculated using the $\left\langle\mathrm{E}_{\text {gas }}\right\rangle$ and $\left\langle\mathrm{G}_{\text {solvation }}\right\rangle$ components.

The crystal structure reveals that the interaction between MDM2 and p53 relies primarily on vdw contacts and steric complementarity. ${ }^{25}$ Residues F19, W23, and L26 of p53 make extensive contacts with MDM2's hydrophobic cleft. For residues of p53, experimental and computational studies have shown that mutants of the hydrophobic residues reduce or eliminate binding, but mutations of polar or charged residues have little effect upon the binding activity. ${ }^{14,35-37}$ We verified our computational approach by calculating the $\Delta \Delta \mathrm{G}_{\text {bind }}$ for p53 mutants T18A, F19A, S20A, and W23A, and comparing the results to previous calculations and experimental values. Our calculated $\Delta \Delta \mathrm{G}_{\text {bind }}$ are $-4.7 \mathrm{kcal} / \mathrm{mol}$ for $\mathrm{W} 23 \mathrm{~A}$ and -3.0 $\mathrm{kcal} / \mathrm{mol}$ for F19A. Values for T18A and S20A are -0.9 $\mathrm{kcal} / \mathrm{mol}$ and $-0.7 \mathrm{kcal} / \mathrm{mol}$. These values parallel Massova and Kollman's calculations $\left(\Delta \Delta \mathrm{G}_{\text {bind }}\right.$ of $-5.54,-2.71$, -0.04 , and $0.72 \mathrm{kcal} / \mathrm{mol}$, respectively). ${ }^{14}$ The results for W23A, F19A, and T18A are within $1 \mathrm{kcal} / \mathrm{mol}$ of Massova and Kollman's results, which is exceptional agreement. Even the S20A values, though different in sign, are well within the typical error bars for this methodology and point to a minimal effect of mutating S20 (less than 1 $\mathrm{kcal} / \mathrm{mol}$ ). The calculated $\Delta \Delta \mathrm{G}_{\text {bind }}$ also agree with the experimental trend that mutants of W23 and F19 abolish p53 binding, while mutations of T18 and S20 are tolerated.

The effects of mutating the residues within MDM2 are not as well understood. Residues of MDM2 with significant influence on the binding (so-called binding hotspots) are important to medicinal chemists for drug-design purposes. In order to determine which residues are most influential, we applied computational alanine scanning to 11 residues 
TABLE II. Relative Free Energies of Binding (kcal/mol) for Alanine Mutants of Human MDM2 Complexed With a 13-residue Helix From the Sequence of Human p53 ${ }^{\text {a }}$

\begin{tabular}{|c|c|c|c|c|}
\hline Contribution & L54A & L57A & $\mathrm{I} 61 \mathrm{~A}$ & M62A \\
\hline$\Delta \Delta \mathrm{E}_{\text {electrostatic }}$ & -1.1 & -0.2 & -0.2 & -0.7 \\
\hline$\Delta \Delta \mathrm{E}_{\mathrm{vdw}}$ & -3.6 & -0.7 & -2.1 & -2.5 \\
\hline$\Delta \Delta \mathrm{E}_{\text {gas }}$ & -4.7 & -0.8 & -2.3 & -3.2 \\
\hline$\Delta \Delta \mathrm{G}_{\text {nonpol }}$ & -0.1 & 0.1 & 0.2 & -0.3 \\
\hline$\Delta \Delta \mathrm{G}_{\mathrm{GB}}$ & 1.2 & 0.1 & -0.6 & 0.7 \\
\hline$\Delta \Delta \mathrm{G}_{\text {solvation }}$ & 1.1 & 0.1 & -0.5 & 0.4 \\
\hline$\Delta \Delta \mathrm{G}_{\text {bind }}$ & $-3.5^{c}$ & -0.7 & -2.7 & -2.8 \\
\hline Interacts with residues of p53 & W23, L26, E28 & W23 & $F 19, W 23$ & F19, S20 \\
\hline Contribution & Y67A & Q72A & F91A & V93A \\
\hline$\Delta \Delta \mathrm{E}_{\text {electrostatic }}$ & -0.9 & -4.4 & 0.0 & -0.1 \\
\hline$\Delta \Delta \mathrm{E}_{\mathrm{vdw}}$ & -2.2 & -2.7 & -0.8 & -2.9 \\
\hline$\Delta \Delta \mathrm{E}_{\text {gas }}$ & -3.1 & -7.1 & -0.8 & -3.0 \\
\hline$\Delta \Delta \mathrm{G}_{\text {nonpol }}$ & 0.1 & -0.3 & 0.2 & 0.0 \\
\hline$\Delta \Delta \mathrm{G}_{\mathrm{GB}}$ & -0.1 & 4.1 & -0.4 & 0.4 \\
\hline$\Delta \Delta \mathrm{G}_{\text {solvation }}$ & -0.1 & 3.8 & -0.1 & 0.4 \\
\hline$\Delta \Delta \mathrm{G}_{\text {bind }}$ & -3.2 & -3.3 & -0.9 & -2.5 \\
\hline Interacts with residues of p53 & F19 & $E 17, T 18, F 19, L 22$ & W23 & $F 19, L 22, W 23$ \\
\hline Contribution & H96A & I99A & Y100A & \\
\hline$\Delta \Delta \mathrm{E}_{\text {electrostatic }}$ & 1.3 & -0.2 & -11.1 & \\
\hline$\Delta \Delta \mathrm{E}_{\mathrm{vdw}}$ & -4.8 & -1.5 & -3.5 & \\
\hline$\Delta \Delta \mathrm{E}_{\text {gas }}$ & -3.5 & -1.7 & -14.6 & \\
\hline$\Delta \Delta \mathrm{G}_{\text {nonpol }}$ & -0.3 & 0.1 & -0.3 & \\
\hline$\Delta \Delta \mathrm{G}_{\mathrm{GB}}$ & 0.4 & 0.3 & 8.4 & \\
\hline$\Delta \Delta \mathrm{G}_{\text {solvation }}$ & 0.1 & 0.4 & 8.2 & \\
\hline$\Delta \Delta \mathrm{G}_{\text {bind }}$ & -3.4 & -1.2 & -6.4 & \\
\hline Interacts with residues of p53 & $L 25, L 26, P 27$ & W23, L26 & P27, N29 & \\
\hline
\end{tabular}

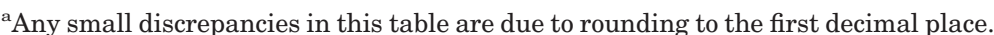

${ }^{\mathrm{b}} \Delta \Delta \mathrm{G}_{\mathrm{bind}}=\Delta \mathrm{G}_{\mathrm{bind}}(\mathrm{WT})-\Delta \mathrm{G}_{\mathrm{bind}}$ (mutant). Negative numbers indicate that the mutation to alanine is unfavorable.

${ }^{c}$ Numbers in bold indicate residues with the most critical contributions $\left(\Delta \Delta \mathrm{G}_{\mathrm{bind}}\right.$ of $-2.5 \mathrm{kcal} / \mathrm{mol}$ or less).

with side chains in contact with p53 and our mimic: L54, L57, I61, M62, Y67, Q72, F91, V93, H96, I99, and Y100. Many of these residues are conserved across different species, ${ }^{14,25}$ and they may be essential for binding p53. The crystal structure of the human p53-MDM2 complex is available ${ }^{25}$ but the structure does not quantify the influence of each residue in terms of ligand binding.

Table II shows the relative free energies of binding for the alanine mutants of the 11 residues of MDM2. The relative free energies of binding are defined as $\Delta \mathrm{G}_{\text {bind }}(\mathrm{WT})-\Delta \mathrm{G}_{\text {bind }}$ (Ala), so negative values for $\Delta \Delta \mathrm{G}_{\text {bind }}$ imply that the mutation to alanine is unfavorable. The results show that there is a significant loss of binding affinity when many of the residues are mutated to alanine. The most detrimental mutation is Y100A, which loses over $6 \mathrm{kcal} / \mathrm{mol}$ of binding free energy. Alanine mutants of L54, Y67, Q72, or H96 cost $3.0-3.5 \mathrm{kcal} / \mathrm{mol}$ of binding free energy. Similarly, the mutation of residues I61, M62, or V93 to alanine is also unfavorable, since the change in $\Delta \mathrm{G}_{\text {bind }}$ is -2.5 to $-3.0 \mathrm{kcal} / \mathrm{mol}$.

As seen in Table II, the replacement of Y100 with alanine was accompanied by a loss of $3.5 \mathrm{kcal} / \mathrm{mol}$ in vdw interactions and $11.1 \mathrm{kcal} / \mathrm{mol}$ in electrostatic energy. During the MD simulations, there are hydrophobic interactions between Y100 and P27 of p53. The stronger electrostatic component above is the result of a hydrogen bond between the phenolic $\mathrm{OH}$ of $\mathrm{Y} 100$ and the $\mathrm{C}$-terminal acid of our p53 helix (the strength of that interaction will be slightly different between the actual complex of p53 and MDM2, because the peptide chain will not terminate at that residue). The mutation of $\mathrm{Q} 72$ to alanine results in a loss of $4.4 \mathrm{kcal} / \mathrm{mol}$ in electrostatic energy and $2.7 \mathrm{kcal} / \mathrm{mol}$ of vdw interaction. During the MD simulation, the sidechain of Q72 forms a hydrogen bond with the backbone nitrogen of F19 in p53.

The major loss when mutating residues L54, I61, M62, Y67, V93, or H96 to alanine is the loss in vdw interaction. Residue L54 of MDM2 packs directly against W23 and L26 of the p53 helix. Though the side chain is critical to the hydrophobic surfaces of the pocket, it is the backbone oxygen of L54 that participates in a hydrogen bond with the indolyl nitrogen of W23 in p53. This hydrogen bond is not lost upon mutating the side chain, so little change is seen in the electrostatic energies. Residues I61 and V93 of MDM2 interact with both F19 and W23 of p53, two critical residues for favorable hydrophobic interaction in the complex. The structures from the MD simulations show that there are favorable vdw interactions between the phenyl ring of Y67 in MDM2 and the phenyl ring of F19 of p53. M62 also has significant vdw interactions with the aromatic ring of F19. MDM2's H96 interacts with P27 and 2 leucine residues in the p53 stretch. Many contacts between 
H96 and p53 indicate that H96 is another binding hotspot, and this is confirmed by a $\Delta \Delta \mathrm{G}_{\text {bind }}$ of $-3.4 \mathrm{kcal} / \mathrm{mol}$.

Residues L57, F91, and I99 do not appear to be as influential for binding as the other hydrophobic residues in the cleft. The smaller contributions from these 3 residues are not obvious from examining the crystal structure of the complex. This underscores the usefulness of the alaninescanning calculations.

Experimental mutagenesis studies have shown G58 to play an important role in binding. The G58 mutants of MDM2 do not interact with p53 in vitro and fail to inhibit p53-dependent transcription in vivo. ${ }^{38}$ Inherent limitations in the alanine-scanning methodology prevent us from quantifying the influence of mutating a glycine, but the MD simulations show tight packing between G58 and the aromatic rings of p53 and our mimic when bound. There was little conformational sampling over the $2 \mathrm{~ns}$ for G58; the average RMSD for residue G58 over 2000 snapshots was only $0.8 \AA$. Mutating G58 to any other residue would likely reduce binding by disrupting the interaction between G58 in MDM2 and the aromatic rings of p53.

These results are in good agreement with experimental results and previous computational studies. Levine and coworkers showed that deletion of residues 43-58, 59-89, or 90-122 from MDM2 eliminated its ability to bind $\mathrm{p} 53 .{ }^{39}$ Leng et al. showed that the deletion of residues $1-58$ or residues 60-65 significantly weakened binding of p53. ${ }^{40}$ These regions encompass the major residues: L54, I61, M62, Y67, Q72, V93, H96, and Y100. These experimental studies support our findings, but that support is limited, since deletion of large sections of the protein alters the structure of MDM2 much more than the alanine mutants presented here. However, our results are in excellent agreement with recent computational studies of Xenopus laevis p53-MDM2 (the binding domain of X. laevis MDM2 has $65 \%$ sequence identity to HMD2).

Kortemme and Baker have recently developed a very interesting approach to alanine-scanning calculations. ${ }^{37}$ In applying their faster method to p53-MDM2 from $X$. laevis, they identified Y67, Q72, V93, and Y100 as hotspots for binding. Our calculations agree with the $X$. laevis data and add L54, I61, M62, and H96 to the list of potential hotspots for human MDM2. Our addition of these 4 residues may come from the differences in our approaches to in silico alanine scanning, or it may be the result of our focus on the human p53-DM2 system. The difference in sequence between $X$. laevis and human MDM2s causes small structural differences that may affect the binding cleft.

\section{Designing a Mimic of p53 Based on $\beta$-Proline}

Given the stability of the complexes in the aforementioned MD simulations and the excellent agreement with experimental free energies of binding, we can assume that these methods provide a reliable means of designing and evaluating potential p53 mimics.

Based on the crystal structure, the MD simulations, and the free energy calculations, we know the importance of hydrophobic complementarity between p53 and MDM2. The alanine-scanning calculations and experimental mutagen- esis studies have shown that F19 and W23 are the most important residues for the binding of p53. Therefore, our mimic must provide aromatic rings to interdigitate MDM2's binding cleft, similar to the side chains of F19 and W23.

An ideal mimic of p53 should consist of (1) a small, helical scaffold that (2) contains side chains similar to phenylalanine and tryptophan, with (3) the proper spacing and orientation to complement MDM2. Short $\beta$-peptides have been shown to fold into helices, sheets, and turns that are common secondary structures in proteins. ${ }^{16-22} \mathrm{We}$ turned to oligomers of $\beta$-proline, $(S)$-pyrrolidine-3-carboxylic acid, as potential scaffolds for the p53 mimic. We have experience calculating the conformational behavior of monomers, dimers, and hexamers of $\beta$-proline. ${ }^{15,16}$ Our ab initio calculations at the RHF/6-31G* level of theory have shown that a $\beta$-proline hexamer can fold into regular helical structures. Both right- and left-handed helices are possible for multimers of $\beta$-proline, making them a good choice for a short helical scaffold. Unsubstituted $\beta$-proline is not rigid in solution, but its conformational freedom is less than a short $\alpha$-peptide, like the p53-based helix used in the modeling and experimental studies discussed above.

Figure 3 shows our process for analyzing the $\beta$-proline helices to design potential mimics of p53 based on the geometric criteria outlined below. In the crystal structure of human p53-MDM2, ${ }^{25}$ the distance between the C $\alpha$ 's of F19 and W23 is $5.9 \AA$; the $\mathrm{C} \alpha-\mathrm{C} \alpha$ distance in the MD simulations of the p53-MDM2 complex remains very close to that value, with an average distance of $6.0 \AA$. In the MD simulations, the RMSDs of all atoms in F19 and W23 are only $1.5 \AA$ and $1.2 \AA$, respectively. These data indicate that the mimic will have to closely match the positions and orientations of F19 and W23. Because the RMSD is a little larger for F19, the benzyl side chain is the one that could be varied a little more in the design of a mimic. The mimic requires side chains that are $5.9 \pm 1.0 \AA$ apart. This distance is based on the crystallographic positions, and the variance is a conservative choice based on the RMSD of W23. The relative orientations of the aromatic side chains are best described by the torsional relationship between $\mathrm{C} \alpha-\mathrm{C} \beta$ in $\mathrm{F} 19$ and $\mathrm{C} \alpha-\mathrm{C} \beta$ in W23. This torsional angle $[\mathrm{C} \beta(\mathrm{F} 19)-\mathrm{C} \alpha(\mathrm{F} 19)-\mathrm{C} \alpha(\mathrm{W} 23)-\mathrm{C} \beta(\mathrm{W} 23)]$ is $16.8^{\circ}$ in the crystal, and the average value in the $\mathrm{MD}$ simulation is $34.7^{\circ}$. Based on these data, the mimic needs side chains that have a torsional relationship of $17 \pm 18^{\circ}$.

The hexamers from our RHF/6-31G* calculations ${ }^{15}$ proved to be too long to fit the cleft for MDM2, but tetramers were approximately the correct length. The 4 lowest energy conformers of the $\beta$-proline helices were analyzed. The conformers of the enantiomer of $\beta$-proline, $(R)$-pyrrolidine-3-carboxylic acid, were also investigated, but successful designs were not possible (data not shown). All pairs of carbons on the $\beta$-proline rings were evaluated as potential substitution points. When pairs of carbons were found that fit the distance criterion (mimicking the two key $\mathrm{C} \alpha$ in $\mathrm{p} 53$ ), their $\mathrm{C}-\mathrm{H}$ bonds were compared to the torsional angle requirement.

After identifying appropriate positions for the aromatic groups, a benzyl side chain (to mimic F19) and an indolyl 


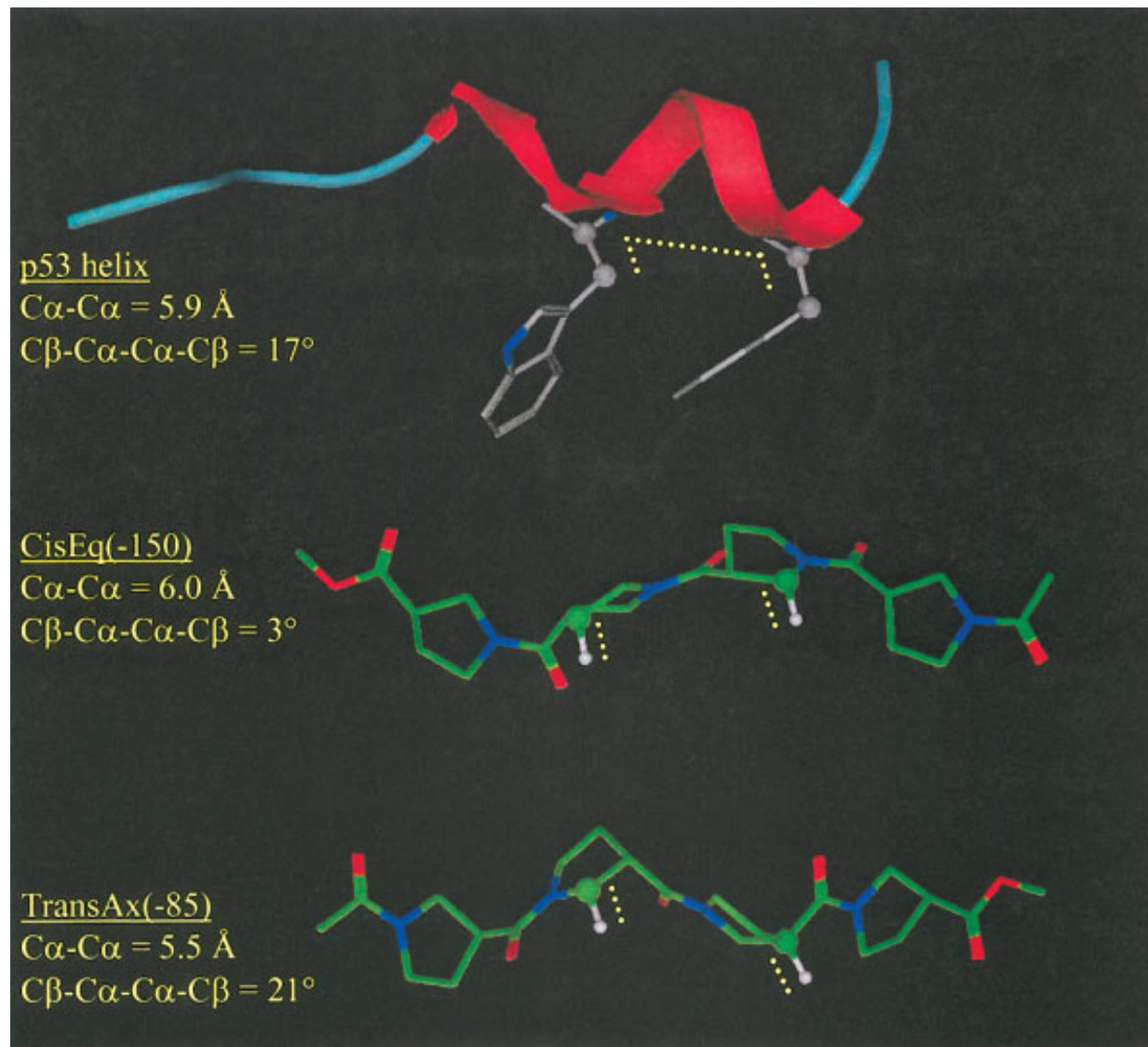

Fig. 3. The evaluation of key residues within $p 53$ to design similar components into the $\beta$-proline scaffold. The $\beta$-proline helices shown with green carbons are from a previous study ${ }^{15}$; these are the lowest energy conformers. Both $\mathrm{N} \rightarrow \mathrm{C}$ and $\mathrm{C} \leftarrow \mathrm{N}$ directions were evaluated for all conformations of the $\beta$-proline scaffold. Within the binding cleft, $\beta$-peptides do not necessarily have to lie in the same direction as $\alpha$-peptides, especially when the p53 helix is capable of internal hydrogen bonding and the mimic is not. The final design of our optimal mimic is based on the middle structure, and it is shown in Figure 4.

moiety (to mimic W23) were introduced into the $\beta$-proline scaffold. The disubstituted tetramers were fit into the MDM2 pocket through superimposing the $\beta$-proline to the p53 sequence according to the $\mathrm{C} \alpha-\mathrm{C} \beta$ bonds of the F19 and W23 side chains. The tetramers and binding-site residues were then energy-minimized to optimize the fit between the mimic and MDM2. Our best p53 mimic based on the $\beta$-proline scaffold is shown in Figure 4; it was chosen based on (1) the relative interaction energy after minimization and (2) the complementarity between the surfaces of the mimic and the MDM2 cleft (data not shown).

The favorable interaction energy is due to the intrinsic hydrophobicity of both surfaces and the ability of the $\beta$-proline scaffold to adopt regular helical structure, presenting the aromatic side chains in the proper orientation. Figure 4 shows the mimic-MDM2 complex superimposed on the p53-MDM2 complex. The mimic appears to be very similar to p53 in terms of the steric complementarities to the hydrophobic cleft and the hydrogen bond between the indolyl nitrogen and the backbone oxygen of L54 in MDM2. No additional group was necessary to mimic L26, another critical hydrophobic residue in p53, because the $\mathrm{N}$-terminal $\beta$-proline ring has favorable interactions with

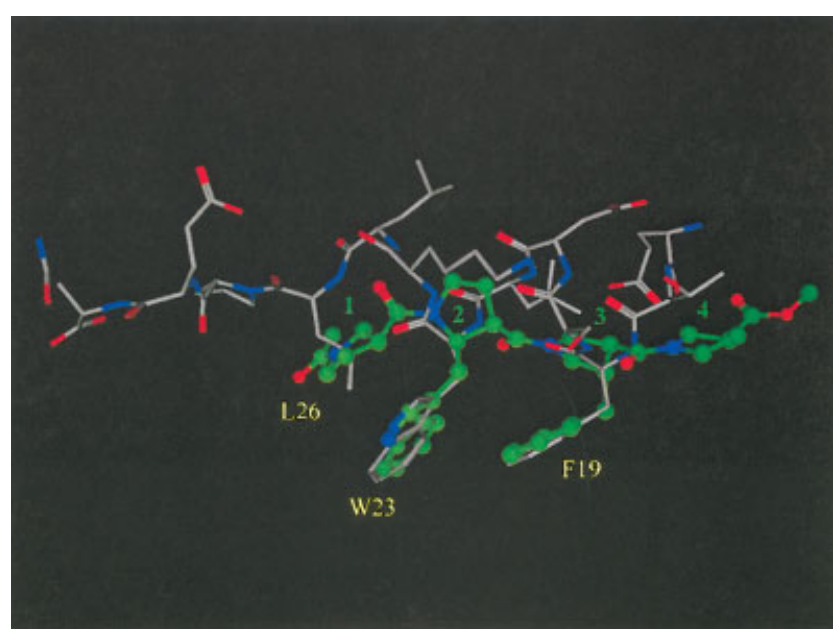

Fig. 4. The similarity between the mimic (ball-and-stick configuration with green atoms) and p53 is shown in an overlay of the complexes of p53-MDM2 ${ }^{25}$ and the mimic-MDM2. The p53 helix has a $\mathrm{C} \leftarrow \mathrm{N}$ orientation in this view, but the optimal fit with the mimic was an $\mathrm{N} \rightarrow \mathrm{C}$ orientation (the side chains are noted in yellow and the $\beta$-proline rings are numbered in green). For clarity, the hydrogens and the protein are not shown. 
A MD of Mimic-MDM2 Complex

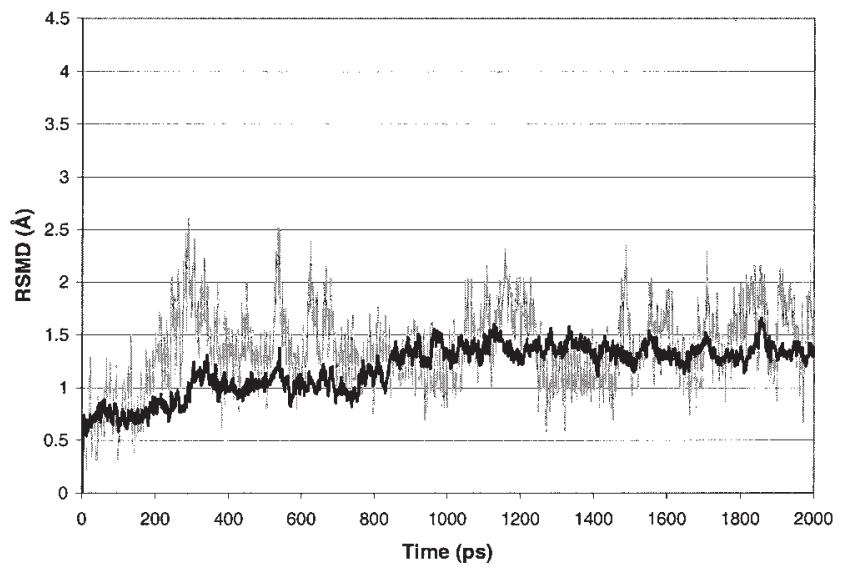

B Independent MD of Unbound Mimic and p53

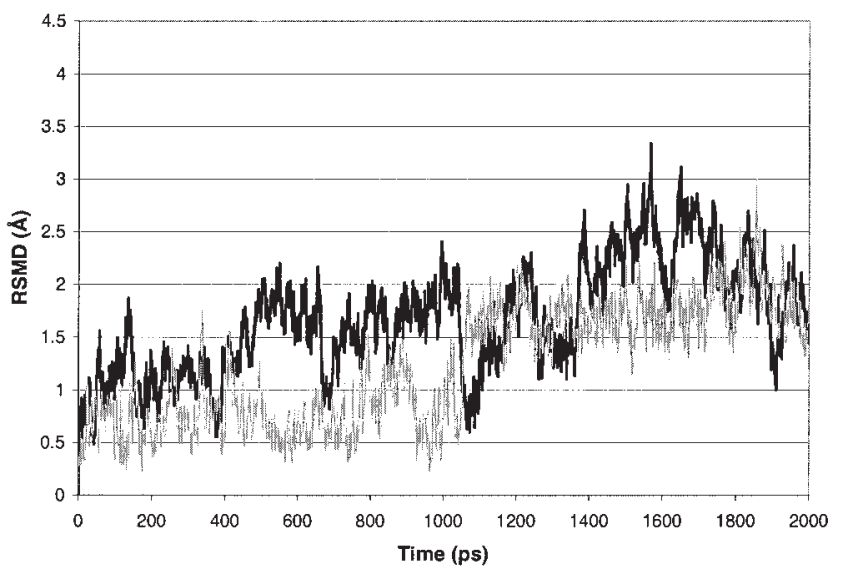

Fig. 5. (A) The RMSDs for the $\mathrm{C} \alpha$ atoms are provided for the mimic-MDM2 complex (thick black line) and the mimic in the complex (thin gray line). (B) The RMSDs for the $\mathrm{C} \alpha$ atoms in the simulations of the unbound mimic (thin gray line); the RMSDs for the unbound p53 (thick black line) are provided for reference.

the binding cleft in the appropriate region. The $\beta$-proline scaffold has an inherently different helical structure, which results in the tetramer having additional interactions with the aromatic ring of Y67 (beyond of the surface complemented by the p53 helix). This does not cause a significant conformational change in the interacting residue of MDM2. In fact, this new hydrophobic interaction contributes additional stability to the complex.

\section{Binding Affinity of the Mimic}

MD simulations were also performed for the mimicMDM2 complex and the unbound mimic. The potential energies over both simulations indicated that the complex and the unbound system were stable (data in supplementary information). The conformational sampling is shown in Figure 5, with plots of RMSD versus time. The initial $600 \mathrm{ps}$ show a period of fluctuation as the mimic-MDM2 complex optimizes its contacts. Over the course of the 2-ns simulation of the complex, the substituted tetramer accurately mimics the positions of p53's F19 and W23; the mimic showed only a small degree of conformational sampling [Fig. 2(C)], with an average RMSD of $0.7 \AA$ for the benzyl side chain, $1.0 \AA$ for the indolyl moiety, and 1.4 $\AA$ for all atoms. However, the unbound tetramer demonstrated a different behavior, with a rise in RMSD halfway through the simulation [gray line in Fig. 5(B)]. The relative stability at the beginning and end of the simulation might suggest 2 major conformations for the unbound mimic, caused by a flip of the aromatic rings and a change in the ring pucker of the third $\beta$-proline ring [Fig. 2(D)]. The calculated RMSD for the same side chains in the simulation of the lone mimic are $5.2 \AA$ and $5.7 \AA$, respectively. The difference between the complex and unbound state indicates that the hydrophobic interaction with the cleft of MDM2 helps to maintain the proper orientations of the aromatic side chains in the complex (this was also the case in the simulations with the $\alpha$-helix of p53). It is interesting that the $\beta$-proline backbone shows little conformational movement in the unbound simulation (RMSD is only $1.2 \AA$ ).

The same GBSA method was applied to the mimic-MDM2 complex, the unbound mimic, and the unbound MDM2 calculated previously. As shown in Table III, the estimated free energy of binding for the mimic was $-8.8 \mathrm{kcal} / \mathrm{mol}$. This indicates that our disubstituted $\beta$-proline-based tetramer could be a potent inhibitor for MDM2, with comparable activity to $\mathrm{p} 53$. The mimic is significantly smaller than p53 but reproduces more than half of the vdw interaction energy. The solvation term is much less of a penalty, which is expected given the hydrophobic nature of the tetramer. Together, these factors produce a free energy of binding that is within error of the value p53 for this method.

Computational alanine scanning was also performed for the same 11 residues of MDM2 within the binding site. Table IV shows that the most significant loss of binding affinity occurs upon mutating Y67 and I61 to alanine. To a lesser extent, I99, L54, M62, and V93 also appear to be potential hotspots for binding. Mutating all of the noted residues to alanine costs a significant amount of vdw interaction energy. Many of the interactions between MDM2 and the mimic are similar to those seen in binding p53. For instance, residue $\mathrm{I} 61$ has favorable hydrophobic interactions with both aromatic side chains of the mimic. This same tight interaction with F19 and W23 in p53 made I61 a key residue for binding the natural ligand, too.

The differences between the mimic and the p53 helix are more interesting, as they may identify residues that are likely to give rise to escape mutants that evade the mimic (Table V). As noted above, mutating Y67 to alanine significantly reduces binding of the mimic. Structures from the MD simulation indicate that the aromatic ring of Y67 stacks underneath the last $\beta$-proline ring of the tetramer, creating close vdw interactions. Though p53 also has vdw interactions with Y67, the degree of contact is much less. Y67 could be a more significant hotspot for our mimic. The other residue that has more influence in binding the mimic versus p53 is I99. The first $\beta$-proline ring of the mimic and its $\mathrm{N}$-terminal acyl group fits tightly against the side chain of I99, reaching more deeply into the 
TABLE III. Energy Components and Free Energy of Binding (kcal/mol) for the Mimic-MDM2 Complex ${ }^{\mathrm{a}}$

\begin{tabular}{|c|c|c|c|c|c|c|c|}
\hline \multirow{2}{*}{$\begin{array}{l}\text { Contributions } \\
(\mathrm{kcal} / \mathrm{mol})\end{array}$} & \multicolumn{2}{|c|}{ Mimic-MDM2 } & \multicolumn{2}{|c|}{ Receptor (MDM2) } & \multicolumn{2}{|c|}{ Ligand (Mimic) } & \multirow[b]{2}{*}{$\Delta$} \\
\hline & Average & std dev & average & $\overline{\text { std dev }}$ & Average & std dev & \\
\hline $\mathrm{E}_{\text {electrostatic }}$ & -2449.7 & 71.6 & -2388.8 & 42.6 & -3.5 & 2.1 & $\overline{-57.4}$ \\
\hline $\mathrm{E}_{\mathrm{vdw}}$ & -351.0 & 14.8 & -322.7 & 14.7 & 6.0 & 3.3 & -34.3 \\
\hline $\mathrm{E}_{\text {internal }}$ & 1576.8 & 26.3 & 1456.4 & 26.5 & 107.8 & 6.5 & 12.6 \\
\hline $\mathrm{E}_{\text {gas }}$ & -1223.8 & 74.8 & -1255.1 & 47.7 & 110.3 & 7.0 & -79.1 \\
\hline $\mathrm{G}_{\text {nonpolar }}$ & 43.0 & 0.8 & 42.5 & 0.6 & 6.7 & 0.3 & -6.2 \\
\hline $\mathrm{G}_{\mathrm{GB}}$ & -1154.5 & 68.1 & -1169.3 & 39.1 & -31.4 & 2.0 & 46.1 \\
\hline $\mathrm{G}_{\text {solvation }}$ & -1111.5 & 67.6 & -1126.8 & 38.8 & -24.7 & 1.9 & 39.9 \\
\hline $\mathrm{E}_{\text {gas }}+\mathrm{G}_{\text {solvation }}$ & -2335.3 & 26.6 & -2381.8 & 26.4 & 85.7 & 6.7 & -39.2 \\
\hline $\begin{array}{l}-\mathrm{TS}^{\mathrm{b}} \\
\Delta \mathrm{G}_{\mathrm{bind}} \mathrm{b}\end{array}$ & -1028.1 & 6.0 & -976.8 & 4.6 & -81.7 & 0.6 & $\begin{array}{r}30.4 \\
-8.8\end{array}$ \\
\hline $\begin{array}{l}-\mathrm{TS}^{\mathrm{c}} \\
\Delta \mathrm{G}_{\mathrm{bind}}^{\mathrm{c}}\end{array}$ & -1028.1 & 6.0 & -971.4 & 4.7 & -80.4 & 1.1 & $\begin{array}{r}23.7 \\
-15.5 \\
\end{array}$ \\
\hline
\end{tabular}

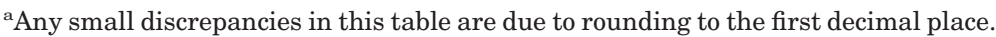

bentropy was estimated based on structures from the trajectory of the complex only. The calculated $\Delta \mathrm{G}_{\text {bind }}$ of -8.8 $\mathrm{kcal} / \mathrm{mol}$ indicates that our proposed disubstituted $\beta$-proline tetramer could be a potent inhibitor for MDM2, with comparable activity to p53.

'Entropy was estimated based on separate trajectories of the complex, MDM2, and the mimic.

TABLE IV. Relative Free Energy of Binding (kcal/mol) for Alanine Mutants of MDM2 Complexed With Our $\beta$-Proline Mimic of $\mathbf{p 5 3}^{\mathrm{a}}$

\begin{tabular}{lrrrr}
\hline Contribution & L54A & L57A & I61A & M62A \\
$\Delta \Delta \mathrm{E}_{\text {electrostatic }}$ & 0.2 & 0.5 & 0.4 & 0.5 \\
$\Delta \Delta \mathrm{E}_{\text {vdw }}$ & -1.9 & -0.7 & -2.0 & -1.9 \\
$\Delta \Delta \mathrm{E}_{\text {gas }}$ & -1.6 & -0.1 & -1.6 & -1.4 \\
$\Delta \Delta \mathrm{G}_{\text {nonpol }}$ & -0.2 & 0.0 & 0.1 & -0.3 \\
$\Delta \Delta \mathrm{G}_{\mathrm{GB}}$ & -0.4 & -0.9 & -1.6 & -0.4 \\
$\Delta \Delta \mathrm{G}_{\text {solvation }}$ & -0.5 & -0.9 & -1.5 & -0.7 \\
$\Delta \Delta \mathrm{G}_{\text {bind }}$ & -2.1 & -1.0 & $-\mathbf{3 . 1}$ & -2.1 \\
& & & & \\
Contribution & $\mathrm{Y} 67 \mathrm{~A}$ & $\mathrm{Q} 72 \mathrm{~A}$ & $\mathrm{~F} 91 \mathrm{~A}$ & $\mathrm{~V} 93 \mathrm{~A}$ \\
$\Delta \Delta \mathrm{E}_{\text {electrostatic }}$ & -0.6 & 0.4 & 0.6 & 0.5 \\
$\Delta \Delta \mathrm{E}_{\text {vdw }}$ & -4.2 & -2.3 & -0.8 & -1.7 \\
$\Delta \Delta \mathrm{E}_{\text {gas }}$ & -4.8 & -1.9 & -0.3 & -1.1 \\
$\Delta \Delta \mathrm{G}_{\text {nonpot }}$ & -0.2 & -0.3 & 0.2 & -0.1 \\
$\Delta \Delta \mathrm{G}_{\mathrm{GB}}$ & -0.3 & 0.7 & -1.0 & -0.7 \\
$\Delta \Delta \mathrm{G}_{\text {solvation }}$ & -0.6 & 0.4 & -0.8 & -0.8 \\
$\Delta \Delta \mathrm{G}_{\text {bind }}$ & $-\mathbf{5 . 3}$ & -1.5 & -1.0 & -1.9 \\
Contribution $_{\Delta \Delta \mathrm{E}_{\text {electrostatic }}}$ & $\mathrm{H} 96 \mathrm{~A}$ & $\mathrm{I} 99 \mathrm{~A}$ & $\mathrm{Y} 100 \mathrm{~A}$ & \\
$\Delta \Delta \mathrm{E}_{\text {vdw }}$ & 0.4 & 0.4 & -0.6 & \\
$\Delta \Delta \mathrm{E}_{\text {gas }}$ & -1.6 & -2.0 & 0.0 & \\
$\Delta \Delta \mathrm{G}_{\text {nonpol }}$ & -1.2 & -1.6 & -0.6 & \\
$\Delta \Delta \mathrm{G}_{\mathrm{GB}}$ & -0.3 & 0.1 & -0.1 & \\
$\Delta \Delta \mathrm{G}_{\text {solvation }}$ & -0.2 & -0.8 & 0.0 & \\
$\Delta \Delta \mathrm{G}_{\text {bind }}$ & -0.5 & -0.7 & -0.1 & \\
\hline
\end{tabular}

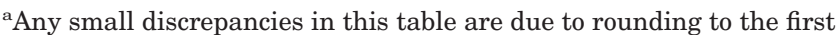
decimal place.

${ }^{\mathrm{b}} \Delta \Delta \mathrm{G}_{\text {bind }}=\Delta \mathrm{G}_{\text {bind }}(\mathrm{WT})-\Delta \mathrm{G}_{\text {bind }}$ (mutant). Negative numbers indicate that the mutation to alanine is unfavorable.

${ }^{\mathrm{c} N u m b e r s}$ in bold indicate residues with the most critical contributions $\left(\Delta \Delta \mathrm{G}_{\text {bind }}\right.$ of $-2.5 \mathrm{kcal} / \mathrm{mol}$ or less $)$.

hydrophobic cleft at that location. This produces greater vdw interaction energies between I99 and the mimic in the subpocket for L26 in p53.
TABLE V. Comparison of Relative Free Energies of Binding (kcal/mol) From the Computational Alanine Scanning for Both Ligands Complexed to Human MDM2

Side Chain

$\begin{array}{llll}\text { in MDM2 } & \text { p53 } & \text { Mimic } & \text { Difference }\end{array}$

L54A

$-3.5$

$-0.7$

$-2.7$

$-2.8$

$-3.2$

-3.3

$-0.9$

$-2.5$

$-3.4$

$-1.2$

$-6.4$

$-21$

$-1.4$

M62A

Q72A

V93A

H96A

Y100A

$-3.1$

$-2.1$

$-5.3$

$-1.5$

$-1.0$

$-1.9$

$-1.6$

$-2.3$

$-0.7$

0.3

0.4

$-0.7$

2.1

$-1.8$

0.1

$-0.6$

$-1.8$

1.1

$-5.7$

aThe greatest differences are highlighted in bold. For Y67A, the mutation is more detrimental to the binding of the mimic. For Q72A, $\mathrm{H} 96 \mathrm{~A}$, and Y100A, the mutation is more detrimental to the binding of the p53 helix.

Some side chains have stronger interactions with p53 than the mimic. Mutations at these positions would be less likely to alter the binding of our mimic without also greatly altering the affinity of p53. For example, Y100 plays an important role when p53 binds to MDM2 through hydrogen bonding to N29 and hydrophobic interactions with P27. These do not apply to the tetramer-MDM2 interaction. Similarly, the side chain of Q72 in MDM2 forms a hydrogen bond with the backbone nitrogen of F19 in p53, but the $\beta$-proline backbone of the mimic has no hydrogen-bond donors to engage in the same strong interaction. Residues H96 and L54 have many vdw contacts with several C-terminal residues of the p53 helix. The vdw interaction between those resi- 

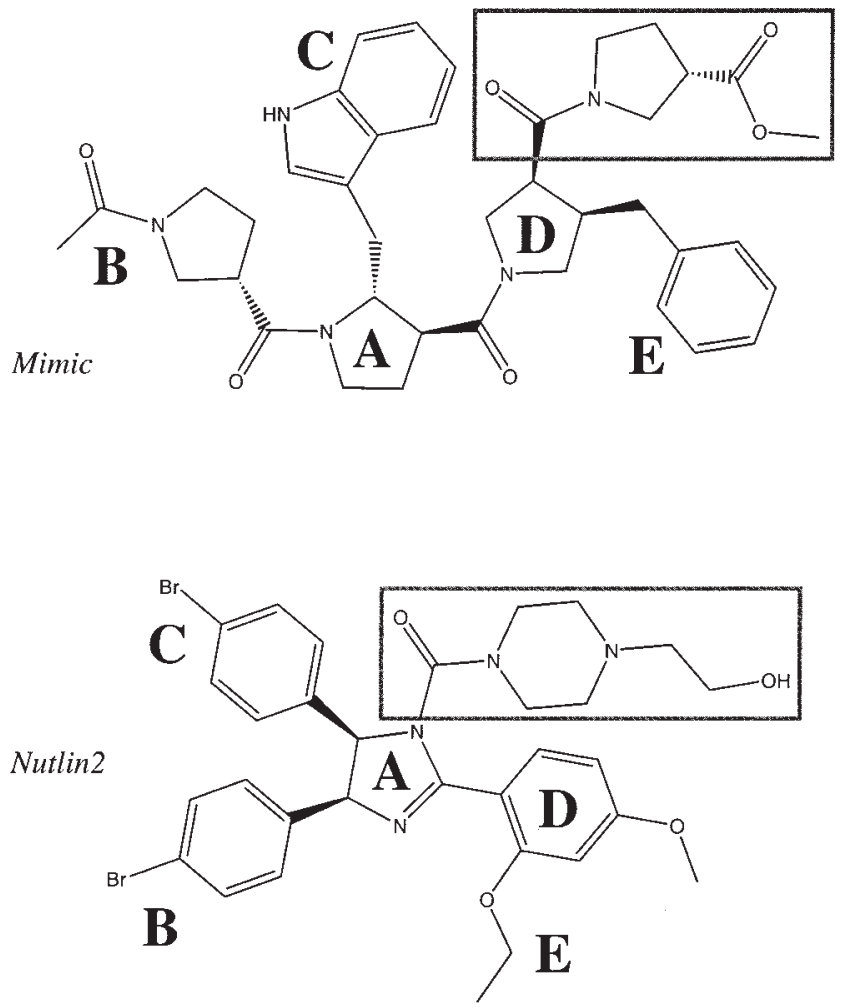

Fig. 6. A comparison of the similar topologies of the mimic and nutlin2. ${ }^{13}$ (A) Central, 5-membered ring. (B) Rings with significant exocyclic groups to play the role of L26 in p53. (C) Aromatic rings play the role of W23. (D) Additional rings present hydrophobic groups (E) to occupy the F19 pocket of MDM2. Regions that are dissimilar are marked with boxes.

dues and the mimic is only moderate because the tetramer is shorter than p53.

\section{Comparing the Mimic to Nutlins}

Of course, the mimic introduced here is meant to be a proof-of-principle exercise to show how one might design peptide mimics to block protein-protein binding and use alanine-scanning calculations to predict escape mutants early in the design process. It was exciting to see similarities to a proven inhibitor of the system. Near the completion of writing this manuscript, nutlins were introduced as a new class of small-molecule inhibitors that block the association of p53-MDM2 ${ }^{13}$ The similarities between the mimic and the nutlins were intriguing, because they were developed in very different ways. The nutlins were discovered through screening diverse libraries of small molecules, and the mimic was a design rationally based on the structural features of the p53-MDM2 complexes from the MD simulations.

Nutlins are also hydrophobic molecules composed of tethered 5- and 6-membered rings, and the topology is rather similar (Fig. 6). The second $\beta$-proline ring of our mimic plays the same role as the central imidazole ring of nutlin2. Both the mimic and nutlin2 use a ring off the central 5-membered ring to occupy the position of L26 in p53. Aromatic rings occupy the position of W23. A third aromatic ring off nutlin2's imidazole ring is a bridging feature, like the third $\beta$-proline ring of our mimic. Both present hydrophobic groups to occupy the position of F19. The piperazine-based branch of nutlin2 is not mirrored in the mimic, and the C-terminal $\beta$-proline ring is not mirrored in nutlin2. However, the structures show that the alcohol group in nutlin 2 is within $1 \AA$ of the nitrogen in the last $\beta$-proline ring. The differences highlighted in Figure 6 result in the mimic being longer than nutlin2, and nutlin2 slightly wider. Figure 7 shows the mimic-MDM2 complex and nutlin2-MDM2 complex overlaid.

\section{Improving Inhibitors of p53-MDM2}

The nutlins are quite a breakthrough in blocking a key protein-protein recognition event, but the crystal structure of the nutlin2-MDM2 complex (1RV1) shows that nutlin2 has unusual packing characteristics. ${ }^{13}$ The nutlin appears to self-associate and also to bind to other faces of MDM2. It is possible that nutlins may be promiscuous binders. The differences between the mimic and the nutlins point to modifications that could be introduced to improve the affinity and specificity of both molecules.

Perhaps the mimic's indolyl ring could be incorporated into the nutlins or groups could be added to lengthen the nutlins to occupy the position of the C-terminal $\beta$-proline ring of the mimic. Additional groups could be introduced off the third $\beta$-proline ring of the mimic to occupy the region of the piperazine-based branch in nutlin2. Also, the nutlins use very different aromatic and hydrophobic groups to complement the pocket, and those groups or other groups could be introduced to the mimic.

Modifying the hydrophobic groups could have an added benefit. During our examination of the crystal structures and the structures from our MD simulations, we discovered a small hydrophobic pocket within MDM2 (Fig. 8). The pocket is rather close to the binding cleft, and it may be possible to design inhibitors to take advantage of it. The pocket is created between L34, L57, L85, F86, I103, and N106. L57 is one of the residues that complements W23 and the indolyl ring of the mimic. In both cases, the L57A mutant gives little change in the free energy of binding. This may indicate that there would be little penalty for L57 to reorient, filling the interior pocket and deepening the binding cleft to allow for larger or differently shaped hydrophobic groups. In the nutlin2-MDM2 structure, ${ }^{13}$ the hydrophobic pocket is larger and includes L37 and L82 as 2 more residues bounding the space. There may be a good deal of flexibility in the hydrophobic residues, and it may also be possible to have a much larger hydrophobic group in place of the indolyl ring that twists its way into the additional space. COX-2 specific inhibitors ${ }^{41,42}$ take advantage of small pockets, and selective estrogen receptor modulators like raloxifene ${ }^{43}$ have very large hydrophobic side chains that wind into hydrophobic interiors of the protein target.

In Table III, the $\Delta \mathrm{G}$ of hydration for the mimic is estimated to be $4.0 \mathrm{kcal} / \mathrm{mol}\left(\mathrm{E}_{\text {gas }}+\mathrm{G}_{\text {solvation }}-\mathrm{TS}\right)$. It is possible that the disubstituted tetramer may have poor solubility for some binding assays, but the hydrophobicity may result in good 


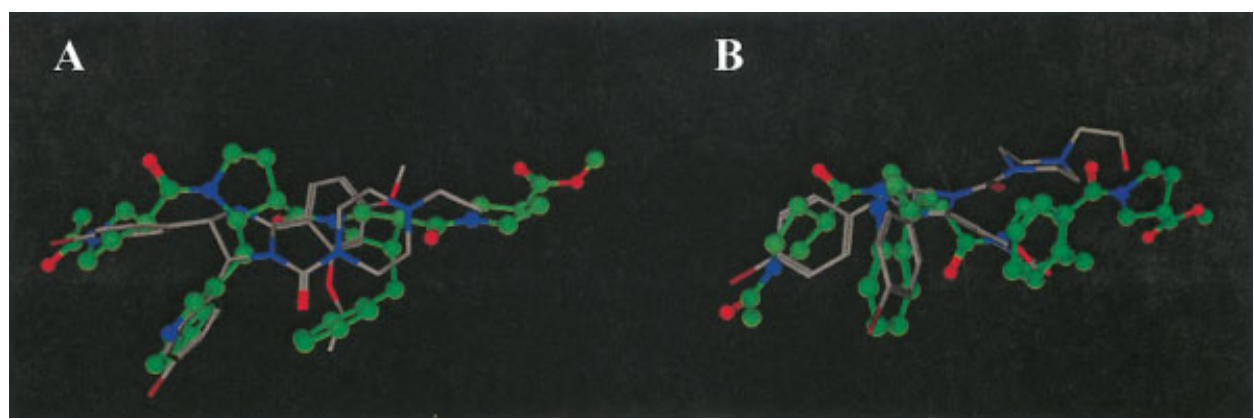

Fig. 7. Two views are used to show the similarity and differences between the mimic (ball-and-stick configuration with green carbons) and nutlin2. This is an overlay of the complexes of nutlin2-MDM2 ${ }^{13}$ and mimic-MDM2, but for clarity, the proteins are not shown.
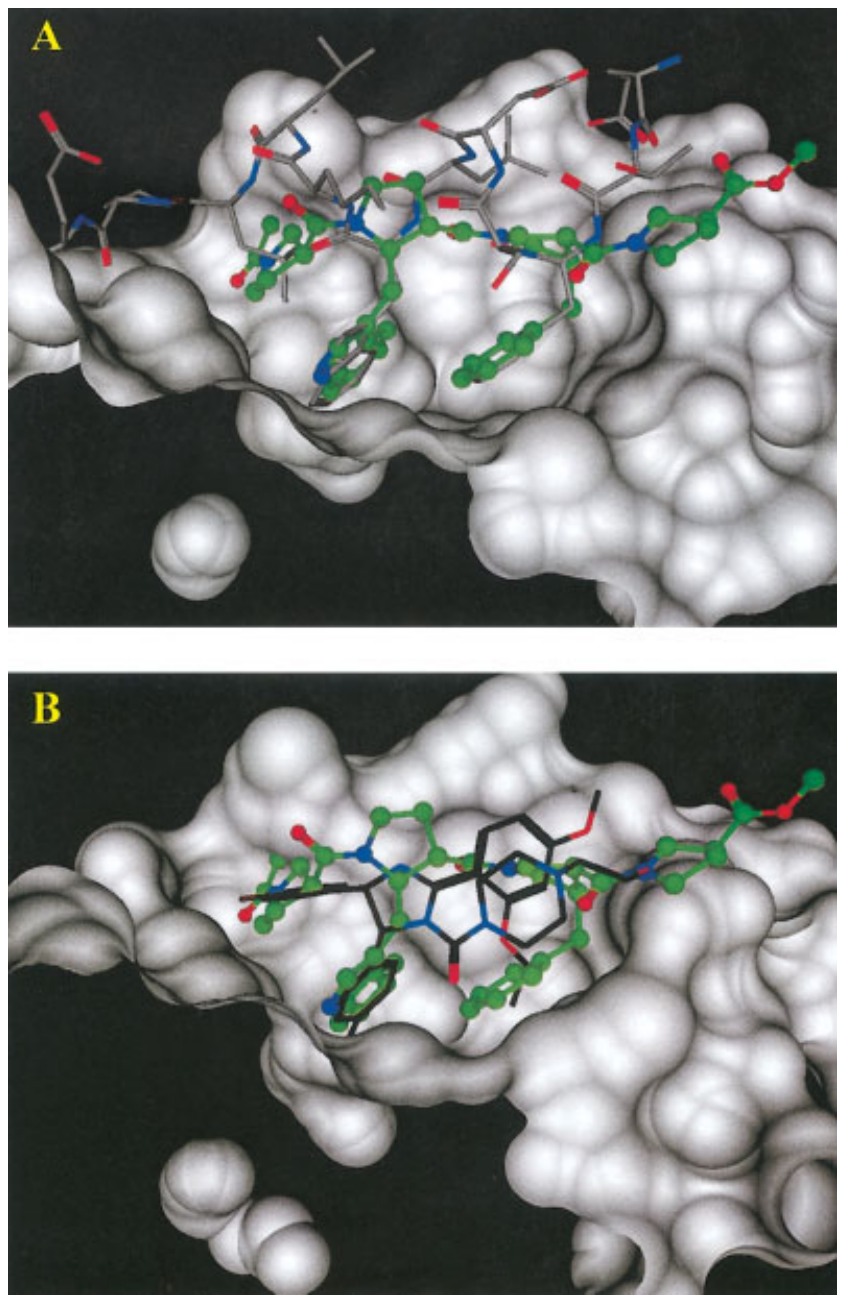

Fig. 8. An additional hydrophobic pocket is available within human MDM2 (lower lefthand quadrant). The pocket is seen within $(\mathbf{A})$ the p53-MDM2 ${ }^{25}$ and (B) the nutlin2-MDM2 ${ }^{13}$ complexes. Its size is variable, and it may be possible to design new inhibitors to take advantage of the additional space within the hydrophobic core.

bioavailability. Functional groups introduced to complement the sites mentioned below could alter the solubility. However, such modifications should be carefully chosen, so that pharmacokinetic properties are also considered.
Further optimization of this mimic could take advantage of additional interactions available in the binding site. Inspection of the snapshots from the MD simulation of the mimic-MDM2 complex reveals potential hydrogen-bonding interactions within the binding cleft that could be used to further improve binding. The backbone oxygen on Q72 does not participate in a hydrogen bond. The side chain of Q72 does form a hydrogen bond to p53, but even with the natural ligand, the backbone oxygen is not complemented. The backbone oxygen of V93 is also available to interact with a ligand, but it is more solvent-exposed. A hydrogen bond between the mimic and the backbone of Q72 or V93 could enhance binding in a manner not seen in p53, and an additional interaction with the backbone of MDM2 should be less prone to contributing to binding hotspots.

\section{CONCLUSIONS}

We have successfully applied the GBSA method to estimate the binding free energy of a p53 helix to MDM2 as -7.4 $\mathrm{kcal} / \mathrm{mol}$. This is in very good agreement with the experiments $(-6.6--8.8 \mathrm{kcal} / \mathrm{mol})$ and implies that we have reliable methodology for modeling this system. We have designed a novel mimic of p53 based on a tetramer of $\beta$-proline, a promising peptidomimetic oligomer. Its estimated free energy of binding is $-8.8 \mathrm{kcal} / \mathrm{mol}$, indicating that this mimic could be a potent inhibitor of MDM2. MD simulations and computational alanine-scanning studies for both p53-MDM2 and the mimic-MDM2 complexes reveal the common binding hotspots could be L54, I61, M62, G58, and V93. Y67 is predicted to be a hotspot for binding both ligands, but it is more significant for the mimic. This makes it possible for Y67 to be the site for escape mutants that evade our mimic. This could be taken into consideration when trying to improve the mimic.

The mimic was compared to nutlin2, a new inhibitor of p53-MDM2 binding. Interesting similarities were found, and comparisons between the 2 structures revealed potential modifications that could be made to improve the mimic and possibly the nutlins. Second-generation designs could take advantage of potential hydrogen-bonding interactions in the cleft or exploit an internal hydrophobic pocket in MDM2. [Plots of the potential energies over the course of the MD simulations and the parameters for the mimic are provided in supplementary materials which can be 
found at http://www.interscience.wiley.com/jpages/08873585/Suppmat/index.html]

\section{NOTE ADDED IN PROOF}

Schepartz and coworkers ${ }^{44}$ have recently created a $\beta$-peptide mimic of p53-MDM2 binding.

\section{ACKNOWLEDGMENTS}

Our thanks to Prof. Samuel H. Gellman (University of Wisconsin, Madison) for helpful discussion and to Mr. Allen Bailey for system administration.

\section{REFERENCES}

1. Kastan MB, Onyekwere O, Sidransky D, Vogelstein B, Craig RW. Participation of p53 protein in the cellular response to DNA damage. Cancer Res 1991;51:6304-6311.

2. Wu X, Levine AJ. p53 and E2F-1 cooperate to mediate apoptosis. Proc Natl Acad Sci USA 1994;91:3602-3606.

3. Hermeking H, Eick D. Mediation of c-Myc-induced apoptosis by p53. Science 1994;265:2091-2093.

4. Oliner JD, Pietenpol JA, Thiagalingam S, Gyuris J, Kinzler KW, Vogelstein B. Oncoprotein MDM2 conceals the activation domain of tumour suppressor p53. Nature 1993;362:857-860.

5. Greenblatt MS, Bennett WP, Hollstein M, Harris CC. Mutations in the p53 tumor suppressor gene: clues to cancer etiology and molecular pathogenesis. Cancer Res 1994;54:4855-4878.

6. Oliner JD, Kinzler KW, Meltzer PS, George DL, Vogelstein B. Amplification of a gene encoding a p53-associated protein in human sarcomas. Nature 1992;358:80-83.

7. Marchetti A, Buttitta F, Girlando S, Dalla Palma P, Pellegrini S, Fina P, Doglioni C, Bevilacqua G, Barbareschi M. MDM2 gene alterations and MDM2 protein expression in breast carcinomas. J Pathol 1995; 175:31-38.

8. Reifenberger G, Liu L, Ichimura K, Schmidt EE, Collins VP. Amplification and overexpression of the MDM2 gene in a subset of human malignant gliomas without p53 mutations. Cancer Res 1993;53:2736-2739.

9. Bueso-Ramos CE, Yang Y, deLeon E, McCown P, Stass SA, Albitar M. The human MDM-2 oncogene is overexpressed in leukemias. Blood 1993;82:2617-2623.

10. Wasylyk C, Salvi R, Argentini M, Dureuil C, Delumeau I, Abecassis J, Debussche L, Wasylyk B. p53 mediated death of cells overexpressing MDM2 by an inhibitor of MDM2 interaction with p53. Oncogene 1999;18:1921-1934.

11. Böttger A, Böttger V, García-Echeverría C, Chène $P$, Hochkeppel HK, Sampson W, Ang K, Howard SF, Picksley SM, Lane DP. Molecular characterization of the HDM2-p53 interaction. J Mol Biol 1997;269:744-756.

12. García-Echeverría C, Chène P, Blommers MJ, Furet P. Discovery of potent antagonists of the interaction between human double minute 2 and tumor suppressor p53. J Med Chem 2000;43:3205-3208.

13. Vassilev LT, Vu BT, Graves B, Carvajal D, Podlaski F, Filipovic Z, Kong N, Kammlott U, Lukacs C, Klein C, Fotouhi N, Liu EA. In vivo activation of the p53 pathway by small-molecule antagonists of MDM2. Science 2004;303:844-848.

14. Massova I, Kollman PA. Computational alanine scanning to probe protein-protein interactions: a novel approach to evaluate binding free energies. J Am Chem Soc 1999;121:8133-8143.

15. Sandvoss LM, Carlson HA. Conformational behavior of $\beta$-proline oligomers. J Am Chem Soc 2003;125:15855-15862.

16. Huck BR, Fisk JD, Guzei ID, Carlson HA, Gellman SH. Secondary structural preferences of 2,2-disubstituted pyrrolidine-4-carboxylic acid oligomers: $\beta$-peptide foldamers that cannot form internal hydrogen bonds. J Am Chem Soc 2003;125:9035-9037.

17. Seebach D, Matthews JL. $\beta$-peptides: a surprise at every turn. Chem Commun (Camb)1997:2015-2022.

18. DeGrado WF, Schneider JP, Hamuro Y. The twists and turns of beta-peptides. J Peptide Res 1999;54:206-217.

19. Cheng RP, Gellman SH, DeGrado WF. $\beta$-peptides: from structure to function. Chem Rev 2001;101:3219-3232.

20. Gellman SH. Foldamers: a manifesto. Acc Chem Res 1998;31:173180 .
21. Kirshenbaum K, Zuckermann RN, Dill KA. Designing polymers that mimic biomolecules. Curr Opin Struct Biol 1999;9:530-535.

22. Hill DJ, Mio MJ, Prince RB, Hughes TS, Moore JS. A field guide to foldamers. Chem Rev 2001;101:3893-4011.

23. Case DA, Pearlman DA, Caldwell JW, Cheatham TE III, Ross WS, Simmerling CL, Darden TA, Merz KM, Stanton RV, Cheng AL, Vincent JJ, Crowley M, Tsui V, Radmer RJ, Duan Y, Pitera J, Massova I, Seibel GL, Singh UC, Weiner PK, Kollman PA. AMBER 6.0. San Francisco: University of California at San Francisco; 1999

24. Cornel WD, Cieplak P, Bayly CI, Gould IR, Merz JKM, Ferguson DM, Spellmeyer DM, Fox T, Caldwell JW, Kollman PA. A second generation force field for the simulation of proteins, nucleic acids and organic molecules. J Am Chem Soc 1995;117:5179-5197.

25. Kussie PH, Gorina S, Marechal V, Elenbaas B, Moreau J, Levine AJ, Pavletich NP. Structure of the MDM2 oncoprotein bound to the p53 tumor suppressor transactivation domain. Science 1996; 274:948-953.

26. Jorgensen WL, Chandrasekhar J, Madura JD, Impey RW, Klein ML. Comparison of simple potential functions for simulating liquid water. J Chem Phys 1983;79:926-935.

27. Molecular Operating Environment, Version MOE 2002.03. Montreal, Canada: Chemical Computing Group, Inc.; 2002.

28. Darden T, York D, Pedersen L. Particle mesh Ewald: an N $\cdot \log (\mathrm{N})$ method for Ewald sums in large systems. J Chem Phys 1993;98: 10089-10092.

29. Bashford D, Case DA. Generalized Born models of macromolecular solvation effects Annu Rev Phys Chem 2000;51:129-152.

30. Tsui V, Case DA. Molecular dynamics simulations of nucleic acids with a Generalized Born solvation model. J Am Chem Soc 2000;122:2489-2498.

31. Jayaram B, Sprous D, Beveridge DL. Solvation free energy of biomacromolecules: parameters for a modified Generalized Born model consistent with the AMBER force field. J Phys Chem B 1998;102:9571-9576.

32. Sanner MF, Olson AJ, Spehner JC. Reduced surface: an efficient way to compute molecular surfaces. Biopolymers 1996;38:305-320.

33. Case DA. Normal-mode analysis of protein dynamics. Curr Opin Struct Biol 1994;4:285-290.

34. Kottalam J, Case DA. Langvin modes of macromolecules: applications to Crambin and DNA hexamers. Biopolymers 1990;29:1409-1421.

35. Picksley SM, Vojtesek B, Sparks A, Lane DP. Immunochemical analysis of the interaction of p53 with MDM2-fine mapping of the MDM2 binding site on p53 using synthetic peptides. Oncogene 1994;9:2523-2529.

36. Portefaix JM, Thebault S, Bourgain-Guglielmetti F, Del Rio M, Granier C, Mani JC, Navarro-Teulon I, Nicolas M, Soussi T, Pau B. Critical residues of epitopes recognized by several anti-p53 monoclonal antibodies correspond to key residues of p53 involved in interactions with the mdm2 protein. J Immunol Methods 2000;244:17-28.

37. Kortemme T, Baker D. A simple physical model for binding energy hot spots in protein-protein complexes. Proc Natl Acad Sci USA 2002;99:14116-14121.

38. Freedman DA, Epstein CB, Roth JC, Levine AJ. A genetic approach to mapping the p53 binding site in the MDM2 protein. Mol Med 1997;3:248-259.

39. Chen J, Marechal V, Levine AJ. Mapping of the p53 and MDM-2 interaction domains. Mol Cell Biol 1993;13:4107-4114.

40. Leng P, Brown DR, Shivakumar CV, Deb S, Deb S. N-terminal 130 amino acids of MDM2 are sufficient to inhibit p53-mediated transcriptional activation. Oncogene 1995;10:1275-1282.

41. Kurumbail RG, Stevens AM, Gierse JK, McDonald JJ, Stegeman RA, Pak JY, Gildehaus D, Miyashiro JM, Penning TD, Seibert K, Isakson PC, Stallings WC. Structural basis for selective inhibition of cyclooxygenase-2 by anti-inflammatory agents. Nature 1996;384: $644-648$.

42. Luong C, Miller A, Barnett J, Chow J, Ramesha C, Browner MF. Flexibility of the NSAID binding site in the structure of human cyclooxygenase-2. Nat Struct Biol 1996;3:927-933.

43. Brzozowski AM, Pike ACW, Dauter Z, Hubbard RE, Bonn T, Engström O, Öhman L, Greene GL, Gustafsson J-Å, Carlquist M. Molecular basis of agonism and antagonismin the oestrogen receptor. Nature 1997;389:753-758.

44. Kritzer JA, Lear JD, Hodsdon ME, Schepartz A. Helical $\beta$-peptide inhibitors of the p53-hDMZ interaction. J Am Chem Soc 2004;126: 9468-9469. 